\title{
SENTINEL 2 AND LANDSAT-8 BANDS SENSITIVITY ANALYSIS FOR MAPPING OF ALKALINE SOIL IN NORTHERN DRY ZONE OF KARNATAKA, INDIA
}

\author{
S. Meti ${ }^{1 *}$, Hanumesh ${ }^{2}$, P.D. Lakshmi², M.S. Nagaraja ${ }^{3}$, V. Shreepad ${ }^{4}$ \\ ${ }^{1}$ Agronomy, DR Office, UHS, Bagalkot, shankarmeti@ gmail.com \\ ${ }^{2}$ M.Sc (Hort) students, Department of Soil Science and Agricultural Chemistry, COH, Bagalkot, \\ ${ }^{3}$ Soil Science, COH, Bagalkot, pathalnag@yahoo.com \\ ${ }^{4}$ Agril. Economics, DE Office, UHS Bagalkot shripadpv@gmail.com
}

Commission III, WG III/10

KEYWORDS: Soil salinity, pH, SAR, Landsat 8, Sentinel 2, Dry lands,

\begin{abstract}
:
Soil salinization is most common land degradation process occurring in deep vertisol of northern dry zone of Karnataka, India. Accurate and high resolution spatial information on salinization can assist policy makers to better target areas for interventions to avoid aggravation of soil degradation process. Digital soil mapping using satellite data has been identified as a potential means of obtaining soil information. This paper focuses on exploring possibility of using new generation medium resolution Landsat-8 and Sentinel-2 satellite data to map alkaline soils of Ramthal irrigation project area in north Karnataka. Surface soil salinity parameters of zone 20 were correlated with reflectance values of different band and band combination and traditional salinity indices and result has indicated that SWIR bands of both satellite showed significant negative correlation with soil $\mathrm{pH}, \mathrm{EC}(\mathrm{r}=-0.39$ to -0.45$)$ whereas visible and NIR bands did not show significant relation. However rationing of SWIR bands with visible blue band has significantly improved the correlation with soil $\mathrm{pH}$ and $\mathrm{EC}(\mathrm{r}=+0.60$ to +0.70$)$. Traditional salinity index based on visible bands failed to show significant correlation with soil parameters. It is interesting to note that SWIR bands alone did not show significant correlation with soil sodicity parameters like exchangeable $\mathrm{Na}$, SAR, RSC but band rationing with blue bands has significantly improved the correlation ( $\mathrm{r}=0.45)$. High resolution soil salinity map was prepared using simple linear regression model and using this map will serve as base map for the policy makers.
\end{abstract}

\section{INTRODUCTION}

Soil salinization is most common land degradation process which occurs in dry lands due to accumulation of sodium or neutral salts in surface. These soils are hungry and thirsty too because of dry climatic condition. Karnataka is the second most drought prone region of India after Rajasthan. For the last several years monsoon continue to be erratic and deficient, causing severe drought. Water is becoming a scarce commodity and plays a very crucial role in agriculture production. So Government of Karnataka is promoting irrigation schemes and gross irrigated area in the state gradually increased from 12.5 percent of net sown area to 32.8 per cent by 2012-13 (http://shodhganga.inflibnet.ac.in/bitstream/ 10603/60160/7/ chapteriv.pdf). Upper Krishna Project (UKP) was launched to irrigate 6.08 lakh ha land in the drought prone districts of Northern Karnataka viz: Kalaburgi, Yadgir, Raichur, Vijayapura and Bagalkot. Deep vertisol are predominant soils of northern dry zone of Karnataka characterized by high $\mathrm{pH}$ and calcareous in nature with calcium and sodium predominant cations in the soil profile. Bringing these soils under irrigation compound the natural process of salinization by bringing more salts from lower profile to top soil through the evaporating water. This will reduce the agricultural production and become

*Corresponding Author unproductive land after some years. So accurate and high resolution spatial information on salinization at regular interval is needed for policy makers to better target areas for interventions to avoid aggravation of soil degradation process, enhance agricultural production and improve livelihood of small farmers over long run. However traditional field survey for delineation and mapping of saline soil is time consuming and expensive and digital soil mapping using satellite data has been identified as alternate technology to address this issue. Previous studies successfully used medium spatial (30m) and spectral resolution satellite data such as Landsat 5, 7 and IRS data to map saline soil (Douaoui et al., 2007; Sumfleth and Duttmann, 2008; Mohamed and Mohamed, 2017; Metternicht and Zinck, 2009). However currently available new generation satellite with increased spatial $(10-20 \mathrm{~m})$ and spectral resolution such as Landsat- 8 and Sentinel-2 satellite data available in public domain offers new opportunities for mapping saline and alkaline soils with improved accuracy. Hence an attempt is being made to explore the possibility of using these satellite data to map alkaline soils of Ramthal irrigation project of UKP in north Karnataka. 


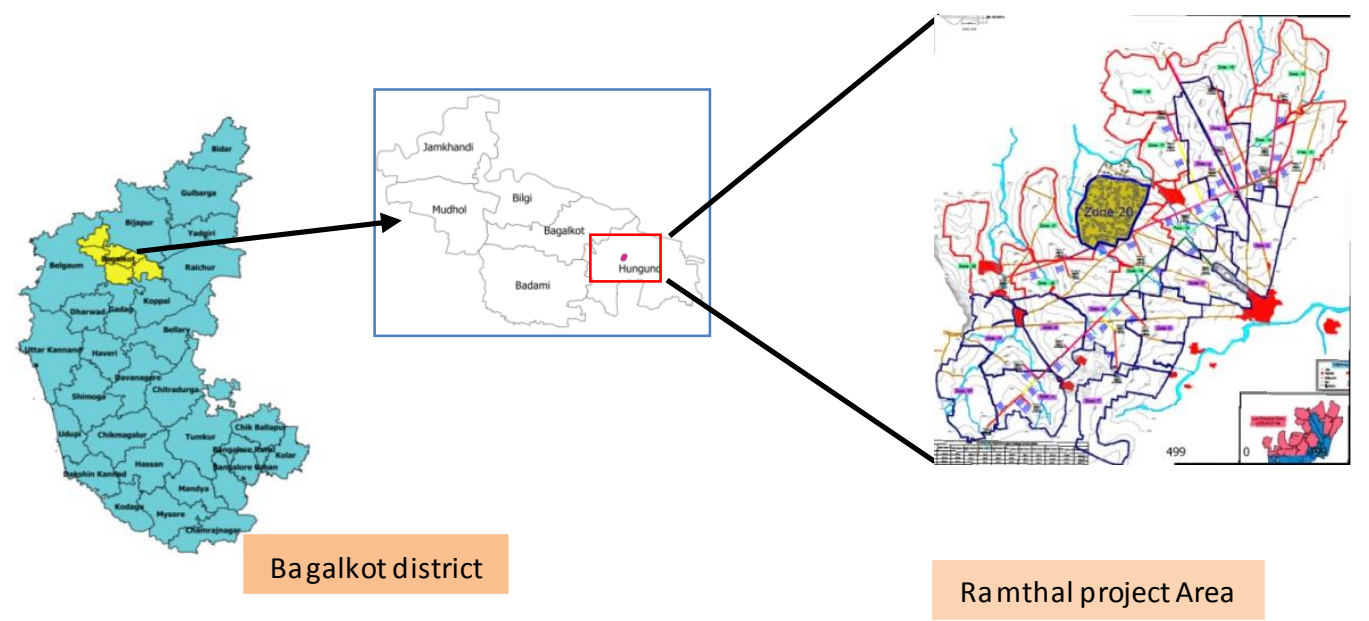

Figure1. Location of study area

\section{METHODS}

\subsection{Study Area}

Asia's largest drip irrigation programme under Stage II of Ramthal (Marol) Lift Irrigation Project was chosen for the study (latitude $16^{0} \mathrm{~N}$ Longitude $76^{\circ} \mathrm{E}$ ). Entire project area covers an area of 11700 ha with elevation ranging from 500 to $550 \mathrm{~m}$ MSL. Based on elevation and slope, total area has been divided into 23 zones of 500ha each with 11 zones in high elevation and 12 zones in low elevation. For soil characterization, contrasting physiographic features are essential and for this zone 20 with elevation variation ranging up to $30 \mathrm{~m}(430-460 \mathrm{~m}$ MSL) was selected for the study.

\subsection{Soil sampling and analysis}

Survey numbers of all the farmers of the study area were collected and soil sample was collected for each land unit representing two hectare. Surface soil sample $(0-15 \mathrm{~cm})$ was collected from three location within two hectare and made into composite sample representing one land unit. Total of 236 surface soil samples $(0-20 \mathrm{~cm})$ spread over zone 20 were collected during April 2017 and recorded geographical location of each sample using hand held GPS. Samples were processed and analyzed for major soil salinity parameters like $\mathrm{pH}$, EC, carbonate and bicarbonate content, cation, anion, $\mathrm{SO}_{4}$, exchangeable sodium, RSC, Sodium Adsorption Ratio (SAR) etc. Soil $\mathrm{pH}$ was determined for 1:2.5 soils: water suspension by using digital $\mathrm{pH}$ meter (Systronics, Model 361) having combined electrode as described by Jackson (1973). The above soil-water suspension (1:2.5) was kept undisturbed for two to four hours and electrical conductivity was measured using conductivity meter (Elico, Model CM 180) and results were expressed in $\mathrm{dS} \mathrm{m}^{-1}$ at $25^{\circ} \mathrm{C}$ (Jackson, 1973). Other parameters were estimated following the standard procedure.

\subsection{Satellite data and processing}

Landsat-8 satellite data corresponding to $05^{\text {th }}$ May 2017 and Sentinel 2 data corresponding to $17^{\text {th }}$ April 2017 covering the study area was downloaded from Earth Explorer website (https://earthexplorer.usgs.gov/). Satellite data were preprocessed and converted each band into top of atmosphere reflectance using Semi-Automatic classification plugin in QGIS. Using GPS reading point, vector file was created and linked the soil analysis data to create geospatial data file of sampling points. Reflectance values for each band of Landsat and Sentinel corresponding to soil sampling point were extracted using point sampling tool in QGIS. Since soil salinization is not a static process, individual bands, the ration between two bands as well as different remote sensing indices such as the salinity index (SI), the normalized difference salinity index (NDSI) etc (Table 1) were investigated for soil salinity mapping. Using reflectance values of different bands, traditional salinity indices as well as all combination of band ratio were calculated and were correlated with soil salinity parameters- $\mathrm{pH}, \mathrm{EC}$, carbonate and bicarbonate content, cation, anion, $\mathrm{SO}_{4}$, exchangeable sodium, RSC, SAR to assess relation between them and identify the best index. Based on the correlation results best index and band ratios were selected. Simple linear regression model with least RMSE was developed using these selected index and band ratios and using this model a high resolution soil salinity map of zone 20 was prepared, which will serve as base map for the policy makers.

Table 1: Salinity indices and their formulae

\begin{tabular}{|c|c|c|}
\hline $\begin{array}{l}\text { Soil salinity } \\
\text { index }\end{array}$ & Formula & Reference \\
\hline Salinity index 1 & $\sqrt{(\text { Blue } x \text { Red })}$ & Khan et al, (2005) \\
\hline Salinity index 2 & $\sqrt{(\text { Green } x \text { Red })}$ & Khan et al, (2005) \\
\hline Salinity index 3 & $\sqrt{(\text { Blue } x ~ S W I R 1)}$ & \\
\hline Salinity index 4 & $\sqrt{(\text { Blue } x \text { SWIR2) }}$ & \\
\hline Salinity index 5 & (Blue x Red)/Green & $\begin{array}{l}\text { Abbas and Khan, } \\
(2007)\end{array}$ \\
\hline Salinity index 6 & (NIR x Red)/Green & $\begin{array}{l}\text { Abbas and Khan, } \\
\text { (2007) }\end{array}$ \\
\hline Salinity index 7 & (SWIR x Red)/Green & \\
\hline Salinity index 8 & $\begin{array}{l}\text { (Blue-SWIR2)/(Blue } \\
+ \text { SWIR2) }\end{array}$ & \\
\hline
\end{tabular}

\section{RESULTS}

Soil of the study area was alkali $(\mathrm{pH}>8.5)$ with low EC $(<0.8$ $\mathrm{ds} / \mathrm{m})$ and dominated with exchangeable cations like calcium (> $\left.32 \mathrm{cmol}\left(\mathrm{p}^{+}\right) \mathrm{kg}^{-1}\right), \mathrm{Mg}\left(>8 \mathrm{cmol}\left(\mathrm{p}^{+}\right) \mathrm{kg}^{-1}\right)$, and $\mathrm{Na}(0.02$ to $\left.9.31 \mathrm{cmol}\left(\mathrm{p}^{+}\right) \mathrm{kg}^{-1}\right)$. Soil solution also showed high soluble salts and carbonates with SAR ranging from 2.9 to 20.7 and RSC -0.2 to 3.5. Average spectral reflectance of five categories 
of soil samples grouped based on $\mathrm{pH}$ and EC indicated distinct difference in reflectance in shortwave infrared (SWIR1) and SWIR 2 and to some extent in Near InfraRed (NIR) only and not so in other bands (Fig. 2\&3). Correlation of satellite bands with soil salinity parameters has indicated that shortwave infrared (SWIR) reflectance of both Landsat 8 and Sentinel 2 satellites showed significant negative correlation with soil $\mathrm{pH}$ and EC (Table 2\&3). Landsat 8 satellite band 6 (SWIR1) and band 7 (SWIR 2) showed significant negative correlation with EC ( $\mathrm{r}=-0.35$ and -0.38$)$ and $\mathrm{pH}(\mathrm{r}=-0.42$ and -0.47$)$ respectively. Similarly sentinel 2 satellite band 11(SWIR1) and band 12 (SWIR 2) reflectance showed significant negative correlation with EC ( $\mathrm{r}=-0.39$ and -0.42$)$ and $\mathrm{pH}(\mathrm{r}=-0.48$ and 0.51 ) respectively. Exchangeable $\mathrm{Na}$ showed significant negative correlation with SWIR and infrared (IR) bands reflectance of Landsat 8 as well as SWIR and NIR bands reflectance of Sentinel 2 satellite. Soil solution Na content also showed significant negative correlation with IR band reflectance but not with NIR band (Band 8) of Semtinel 2 . Calcium and magnesium content in soil solution showed significant positive correlation whereas SAR and total cation in soil solution negative correlation with SWIR bands of both satellite. Visible and near infrared (NIR) bands did not show significant relation with soil salinity parameters (Table 2 and 3).

Rationing of SWIR bands with visible blue and green bands of both satellites has significantly improved the correlation with soil salinity parameters. Among different band ratios, ratio between blue band and SWIR has shown significant positive correlation with $\mathrm{pH}, \mathrm{EC}$ and exchangeable $\mathrm{Na}$ (Table 4 and 5). It is interesting to note that SWIR bands alone did not show significant correlation with soil sodicity parameters like exchangeable $\mathrm{Na}$, SAR, RSC but band rationing with blue bands has significantly improved the correlation $(r=>0.65)$. Majority of traditional salinity index calculated for both satellite based on visible bands failed to show significant correlation with soil salinity parameters. Among the traditional salinity indices, only SI7 and SI8 of both Landsat8 and Sentinel 2 showed better correlation with $\mathrm{pH}, \mathrm{EC}$, exchangeable $\mathrm{Na}$ and other soil salinity parameters (Table 6\&7). Simple linear regression model with least RMSE was developed for
$\mathrm{pH}$, EC and exchangeable $\mathrm{Na}$ (Table 8). Irrespective of satellite, Regression model for $\mathrm{pH}$ and Exchangeable $\mathrm{Na}$ showed higher coefficient $\left(\mathrm{R}^{2}=0.5\right)$ compared to $\mathrm{EC}\left(\mathrm{R}^{2}=0.3\right)$. Reflectance from NIR and SWIR bands contributed significantly to prediction of soil $\mathrm{pH}$ and $\mathrm{Na}$ content.

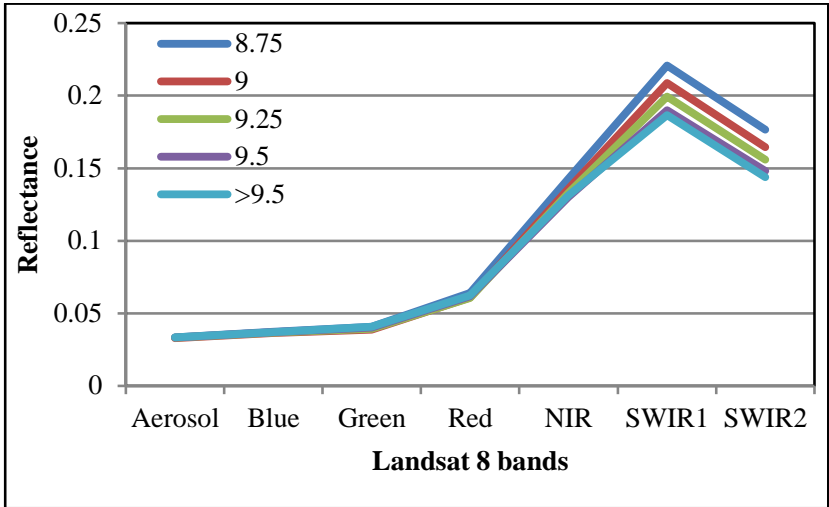

Fig2. Spectral response of different categories of saline soils

based on $\mathrm{pH}$

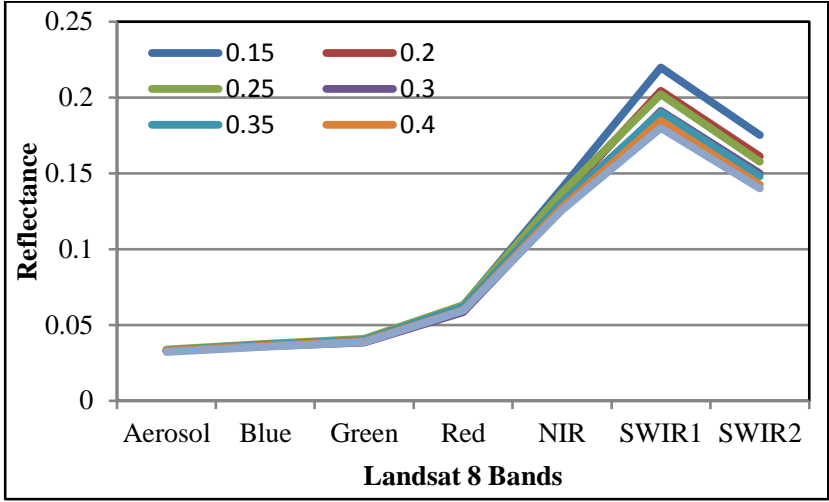

Fig3. Spectral response of different categories of saline soils

based on EC'

Table 2: Correlation between Landsat 8 satellite bands reflectance and soil salinity parameters

\begin{tabular}{|c|c|c|c|c|c|c|c|c|c|c|}
\hline \multirow[b]{2}{*}{ Parameters } & \multicolumn{10}{|c|}{ Landsat 8 bands } \\
\hline & $\begin{array}{c}\text { Band1 } \\
\text { Aerosol }\end{array}$ & $\begin{array}{c}\text { Band2 } \\
\text { Blue }\end{array}$ & $\begin{array}{l}\text { Band3 } \\
\text { Green }\end{array}$ & $\begin{array}{c}\text { Band } 4 \\
\text { Red }\end{array}$ & $\begin{array}{c}\text { Band 5 } \\
\text { NIR }\end{array}$ & $\begin{array}{l}\text { Band } 6 \\
\text { SWIR1 }\end{array}$ & $\begin{array}{l}\text { Band } 7 \\
\text { SWIR2 }\end{array}$ & $\begin{array}{c}\text { Band } 8 \\
\text { PAN }\end{array}$ & $\begin{array}{c}\text { Band } 10 \\
\text { IR1 }\end{array}$ & $\begin{array}{c}\text { Band } 11 \\
\text { IR2 }\end{array}$ \\
\hline $\mathrm{EC}$ & -0.02 & -0.03 & -0.01 & -0.05 & -0.16 & $-0.35^{*}$ & -0.38 & -0.03 & -0.15 & -0.12 \\
\hline $\mathrm{pH}$ & 0.01 & -0.01 & -0.01 & -0.06 & -0.20 & -0.42 & -0.47 & -0.03 & -0.16 & -0.13 \\
\hline Exch $\mathrm{Ca}$ & 0.09 & 0.09 & 0.10 & 0.13 & 0.18 & 0.27 & 0.29 & 0.12 & -0.03 & -0.04 \\
\hline Exch Mg & 0.04 & 0.04 & 0.04 & 0.02 & 0.00 & -0.08 & -0.12 & 0.05 & -0.07 & -0.07 \\
\hline Exch_Na & 0.00 & -0.03 & -0.02 & -0.07 & -0.20 & -0.42 & -0.47 & -0.04 & -0.31 & -0.28 \\
\hline Soil soln-Ca & 0.06 & 0.07 & 0.08 & 0.10 & 0.15 & 0.24 & 0.25 & 0.05 & 0.04 & 0.04 \\
\hline Soil soln $\mathrm{Mg}$ & -0.03 & -0.03 & -0.01 & 0.01 & 0.08 & 0.24 & 0.28 & -0.02 & 0.13 & 0.11 \\
\hline Soil soln $\mathrm{Na}$ & -0.04 & -0.04 & -0.04 & -0.08 & -0.15 & -0.30 & -0.33 & -0.03 & -0.17 & -0.16 \\
\hline Soil soln carbonates & 0.04 & 0.04 & 0.05 & 0.05 & 0.00 & -0.06 & -0.05 & 0.08 & -0.09 & -0.07 \\
\hline Soil soln Cation & -0.04 & -0.04 & -0.04 & -0.07 & -0.13 & -0.25 & -0.28 & -0.02 & -0.16 & -0.15 \\
\hline Soil soln Anion & 0.03 & 0.02 & 0.01 & -0.02 & -0.09 & -0.17 & -0.20 & 0.05 & -0.16 & -0.16 \\
\hline $\mathrm{ECe}$ & 0.06 & 0.05 & 0.06 & 0.03 & -0.05 & -0.15 & -0.18 & 0.03 & -0.24 & -0.21 \\
\hline RSC & 0.02 & 0.02 & 0.02 & 0.00 & -0.08 & -0.21 & -0.22 & 0.05 & -0.13 & -0.11 \\
\hline SAR & -0.05 & -0.06 & -0.06 & -0.10 & -0.18 & -0.34 & -0.38 & -0.04 & -0.18 & -0.17 \\
\hline
\end{tabular}

* Significant at $\mathrm{p}=0.05$ 
Table 3: Correlation between Sentinel 2 satellite bands reflectance and soil salinity parameters

\begin{tabular}{|l|c|c|c|c|c|c|c|c|c|c|}
\hline \multirow{4}{*}{ Parameters } & \multicolumn{9}{|c|}{ Sentinel 2 bands } \\
\cline { 2 - 14 } & $\begin{array}{c}\text { Band1 } \\
\text { Aerosol }\end{array}$ & $\begin{array}{c}\text { Band2 } \\
\text { Blue }\end{array}$ & $\begin{array}{c}\text { Band3 } \\
\text { Green }\end{array}$ & $\begin{array}{c}\text { Band 4 } \\
\text { Red }\end{array}$ & $\begin{array}{c}\text { Band 5 } \\
\text { Rededge }\end{array}$ & $\begin{array}{c}\text { Band 6 } \\
\text { Rededge }\end{array}$ & $\begin{array}{c}\text { Band 7 } \\
\text { Rededge }\end{array}$ & $\begin{array}{c}\text { Band 8 } \\
\text { NIR }\end{array}$ & $\begin{array}{c}\text { Band 11 } \\
\text { SWIR1 }\end{array}$ & $\begin{array}{c}\text { Band 12 } \\
\text { SWIR2 }\end{array}$ \\
\hline EC & -0.14 & -0.11 & -0.08 & -0.12 & -0.13 & -0.16 & -0.17 & -0.20 & $\mathbf{- 0 . 3 9 *}$ & $\mathbf{- 0 . 4 2}$ \\
\hline pH & -0.15 & -0.08 & -0.08 & -0.13 & -0.18 & -0.21 & $-\mathbf{- 0 . 2 5}$ & $\mathbf{- 0 . 2 4 *}$ & $\mathbf{- 0 . 4 8}$ & $\mathbf{- 0 . 5 1}$ \\
\hline Exch_Ca & 0.19 & 0.17 & 0.14 & 0.18 & 0.20 & 0.22 & 0.22 & 0.22 & $\mathbf{0 . 3 2}$ & $\mathbf{0 . 3 3}$ \\
\hline Exch_Mg & 0.02 & 0.01 & 0.01 & 0.00 & -0.02 & -0.04 & -0.05 & -0.02 & -0.12 & -0.15 \\
\hline Exch_Na & -0.18 & -0.11 & -0.09 & -0.13 & -0.18 & -0.21 & $-\mathbf{0 . 2 4}$ & $\mathbf{- 0 . 2 4}$ & $\mathbf{- 0 . 4 9}$ & $\mathbf{- 0 . 5 3}$ \\
\hline Soil Soln_Ca & 0.11 & 0.09 & 0.09 & 0.13 & 0.14 & 0.15 & 0.15 & 0.17 & 0.25 & $\mathbf{0 . 2 7}$ \\
\hline Soil Soln_Mg & 0.05 & 0.04 & 0.00 & 0.04 & 0.06 & 0.07 & 0.09 & 0.10 & 0.27 & $\mathbf{0 . 3 1}$ \\
\hline Soil Soln K & 0.09 & 0.04 & 0.06 & 0.07 & 0.13 & 0.15 & 0.16 & 0.13 & 0.13 & 0.11 \\
\hline Soil Soln Na & -0.11 & -0.06 & -0.04 & -0.07 & -0.10 & -0.10 & -0.11 & -0.14 & -0.30 & $\mathbf{- 0 . 3 5}$ \\
\hline Soil Soln Carbonates & 0.01 & 0.06 & 0.07 & 0.06 & 0.03 & 0.03 & 0.02 & 0.03 & -0.07 & -0.08 \\
\hline Soil Soln Cation & -0.09 & -0.05 & -0.03 & -0.05 & -0.07 & -0.07 & -0.08 & -0.11 & $\mathbf{- 0 . 2 5}$ & $\mathbf{- 0 . 2 9}$ \\
\hline Soil Soln Anion & -0.01 & 0.03 & 0.02 & 0.01 & -0.02 & -0.02 & -0.04 & -0.04 & -0.18 & -0.22 \\
\hline ECe & -0.04 & -0.02 & -0.01 & -0.04 & -0.06 & -0.08 & -0.10 & -0.10 & -0.20 & -0.21 \\
\hline RSC & -0.05 & 0.00 & 0.02 & -0.01 & -0.04 & -0.05 & -0.06 & -0.06 & $\mathbf{- 0 . 2 4}$ & $\mathbf{- 0 . 2 7}$ \\
\hline SAR & -0.14 & -0.09 & -0.07 & -0.11 & -0.13 & -0.14 & -0.15 & -0.18 & $\mathbf{- 0 . 3 6}$ & $\mathbf{- 0 . 4 0}$ \\
\hline
\end{tabular}

* Significant at $\mathrm{p}=0.05$

Table 4: Correlation between Landsat 8 satellite bands ratios and soil salinity parameters

\begin{tabular}{|l|l|l|l|l|l|l|l|l|}
\hline \multirow{2}{*}{ Parameters } & \multicolumn{9}{|c|}{ Landsat8 bands ratios } \\
\cline { 2 - 9 } & B1/B6 & B1/B7 & B2/B5 & B2/B6 & B2/B7 & B3/B5 & B3/B6 & B3/B7 \\
\hline EC & $\mathbf{0 . 5 2} *$ & $\mathbf{0 . 5 3}$ & 0.33 & $\mathbf{0 . 5 1}$ & $\mathbf{0 . 5 0}$ & $\mathbf{0 . 3 4}$ & $\mathbf{0 . 3 8}$ & $\mathbf{0 . 3 8}$ \\
\hline pH & $\mathbf{0 . 6 5}$ & $\mathbf{0 . 6 7}$ & $\mathbf{0 . 4 6}$ & $\mathbf{0 . 6 5}$ & $\mathbf{0 . 6 4}$ & $\mathbf{0 . 4 2}$ & $\mathbf{0 . 4 4}$ & $\mathbf{0 . 4 6}$ \\
\hline Exch_Ca & $\mathbf{- 0 . 3 3}$ & $\mathbf{- 0 . 3 4}$ & -0.28 & $\mathbf{- 0 . 3 0}$ & $\mathbf{- 0 . 3 0}$ & -0.10 & -0.12 & -0.14 \\
\hline Exch_Mg & 0.15 & 0.18 & 0.09 & 0.18 & 0.20 & 0.10 & 0.14 & 0.16 \\
\hline Exch_Na & $\mathbf{0 . 6 5}$ & $\mathbf{0 . 6 7}$ & $\mathbf{0 . 4 2}$ & $\mathbf{0 . 6 3}$ & $\mathbf{0 . 6 3}$ & $\mathbf{0 . 4 0}$ & $\mathbf{0 . 4 4}$ & $\mathbf{0 . 4 6}$ \\
\hline Soil Soln_Ca & $\mathbf{- 0 . 3 0}$ & $\mathbf{- 0 . 3 0}$ & -0.25 & -0.28 & -0.27 & -0.13 & -0.13 & -0.14 \\
\hline Soil Soln_Mg & $\mathbf{- 0 . 4 1}$ & $\mathbf{- 0 . 4 3}$ & -0.27 & $\mathbf{- 0 . 4 1}$ & $\mathbf{- 0 . 4 2}$ & -0.20 & -0.27 & -0.29 \\
\hline Soil Soln_Na & $\mathbf{0 . 4 1}$ & $\mathbf{0 . 4 2}$ & 0.29 & $\mathbf{0 . 4 0}$ & $\mathbf{0 . 4 0}$ & 0.22 & 0.25 & 0.26 \\
\hline Soil Soln_Carbonate & 0.13 & 0.12 & 0.07 & 0.16 & 0.14 & 0.15 & 0.16 & 0.14 \\
\hline Soil Soln_Cation & $\mathbf{0 . 3 3}$ & $\mathbf{0 . 3 4}$ & 0.23 & $\mathbf{0 . 3 3}$ & $\mathbf{0 . 3 3}$ & 0.19 & 0.21 & 0.22 \\
\hline Soil Soln_Anion & $\mathbf{0 . 3 0}$ & $\mathbf{0 . 3 0}$ & 0.24 & $\mathbf{0 . 3 0}$ & 0.29 & 0.22 & 0.20 & 0.20 \\
\hline ECe & $\mathbf{0 . 3 0}$ & $\mathbf{0 . 3 1}$ & 0.20 & $\mathbf{0 . 3 1}$ & 0.31 & 0.27 & 0.26 & 0.27 \\
\hline RSC & $\mathbf{0 . 3 4}$ & $\mathbf{0 . 3 4}$ & 0.23 & $\mathbf{0 . 3 6}$ & $\mathbf{0 . 3 5}$ & 0.23 & 0.26 & 0.26 \\
\hline SAR & $\mathbf{0 . 4 7}$ & $\mathbf{0 . 4 8}$ & $\mathbf{0 . 3 4}$ & $\mathbf{0 . 4 6}$ & $\mathbf{0 . 4 6}$ & 0.23 & 0.27 & 0.29 \\
\hline
\end{tabular}

Table 5: Correlation between Sentinel 2 satellite bands ratios and soil salinity parameters

\begin{tabular}{|l|c|c|c|c|c|c|}
\hline \multirow{2}{*}{ Parameters } & \multicolumn{7}{|c|}{ Sentinel 2 bands ratios } \\
\cline { 2 - 7 } & B1/B11 & B1/B12 & B2/B7 & B2/B8 & B2/B11 & B2/B12 \\
\hline EC & $\mathbf{0 . 5 0} *$ & $\mathbf{0 . 5 1}$ & 0.17 & $\mathbf{0 . 2 9}$ & $\mathbf{0 . 3 7}$ & $\mathbf{0 . 3 6}$ \\
\hline pH & $\mathbf{0 . 6 1}$ & $\mathbf{0 . 6 3}$ & $\mathbf{0 . 3 8}$ & $\mathbf{0 . 4 4}$ & $\mathbf{0 . 5 2}$ & $\mathbf{0 . 5 0}$ \\
\hline Exch_Ca & $\mathbf{- 0 . 3 0}$ & $\mathbf{- 0 . 3 0}$ & -0.21 & -0.24 & -0.15 & -0.15 \\
\hline Exch_Mg & 0.20 & 0.22 & 0.12 & 0.09 & 0.15 & 0.16 \\
\hline Exch_Na & $\mathbf{0 . 6 1}$ & $\mathbf{0 . 6 4}$ & $\mathbf{0 . 3 2}$ & $\mathbf{0 . 4 1}$ & $\mathbf{0 . 5 1}$ & $\mathbf{0 . 5 0}$ \\
\hline Soil Soln_Ca & $\mathbf{- 0 . 2 8}$ & $\mathbf{- 0 . 2 9}$ & -0.18 & -0.27 & -0.19 & -0.19 \\
\hline Soil Soln_Mg & $\mathbf{- 0 . 4 0}$ & $\mathbf{- 0 . 4 2}$ & -0.13 & -0.19 & $\mathbf{- 0 . 3 0}$ & $\mathbf{- 0 . 3 1}$ \\
\hline Soil Soln_K & -0.11 & -0.09 & -0.28 & -0.26 & -0.13 & -0.10 \\
\hline Soil Soln_Na & $\mathbf{0 . 3 7}$ & $\mathbf{0 . 4 0}$ & 0.14 & 0.24 & $\mathbf{0 . 3 1}$ & $\mathbf{0 . 3 2}$ \\
\hline Soil Soln_Carbonates & 0.14 & 0.14 & 0.05 & 0.05 & 0.20 & 0.19 \\
\hline Soil Soln_Cation & $\mathbf{0 . 2 9}$ & $\mathbf{0 . 3 3}$ & 0.10 & 0.19 & 0.26 & 0.27 \\
\hline Soil Soln_Anion & $\mathbf{0 . 2 9}$ & $\mathbf{0 . 3 1}$ & 0.17 & 0.19 & $\mathbf{0 . 3 0}$ & $\mathbf{0 . 3 1}$ \\
\hline ECe & $\mathbf{0 . 2 9}$ & $\mathbf{0 . 3 0}$ & 0.16 & 0.20 & 0.25 & 0.25 \\
\hline RSC & $\mathbf{0 . 3 4}$ & $\mathbf{0 . 3 6}$ & 0.14 & 0.19 & $\mathbf{0 . 3 3}$ & $\mathbf{0 . 3 2}$ \\
\hline SAR & $\mathbf{0 . 4 3}$ & $\mathbf{0 . 4 6}$ & 0.18 & $\mathbf{0 . 3 0}$ & $\mathbf{0 . 3 4}$ & $\mathbf{0 . 3 5}$ \\
\hline
\end{tabular}

* Significant at $\mathrm{p}=0.05$ 
Table 6: Correlation between salinity index calculated based on Landsat 8 bands and soil salinity parameters

\begin{tabular}{|l|l|l|l|l|l|l|l|l|}
\hline \multirow{2}{*}{ Parameters } & \multicolumn{7}{|c|}{ Salinity index based on Landsat8 bands } \\
\cline { 2 - 10 } & SI1 & SI2 & SI3 & SI4 & SI5 & SI6 & SI7 & SI8 \\
\hline EC & -0.04( & -0.03 & -0.21 & -0.24 & -0.09 & -0.19 & $\mathbf{- 0 . 3 8} *$ & $\mathbf{0 . 5 0}$ \\
\hline pH & -0.04 & -0.03 & -0.24 & -0.28 & -0.09 & -0.25 & $\mathbf{- 0 . 4 6}$ & $\mathbf{0 . 6 4}$ \\
\hline Exch_Ca & 0.12 & 0.12 & 0.20 & 0.21 & 0.13 & 0.20 & 0.29 & $\mathbf{- 0 . 3 0}$ \\
\hline Exch_Mg & 0.03 & 0.03 & -0.02 & -0.04 & 0.01 & -0.03 & -0.11 & 0.20 \\
\hline Exch_Na & -0.05 & -0.04 & -0.25 & -0.29 & -0.11 & -0.25 & $\mathbf{- 0 . 4 7}$ & $\mathbf{0 . 6 2}$ \\
\hline Soil Soln_Ca & 0.09 & 0.09 & 0.17 & 0.18 & 0.11 & 0.17 & 0.26 & -0.28 \\
\hline Soil Soln_Mg & 0.00 & 0.00 & 0.12 & 0.15 & 0.02 & 0.11 & 0.26 & $\mathbf{- 0 . 4 2}$ \\
\hline Soil Soln_Na & -0.07 & -0.06 & -0.19 & -0.21 & -0.10 & -0.19 & $\mathbf{- 0 . 3 3}$ & $\mathbf{0 . 4 0}$ \\
\hline Soil Soln_Carbonate & 0.05 & 0.05 & -0.01 & -0.01 & 0.04 & -0.01 & -0.06 & 0.14 \\
\hline Soil Soln_Cation & -0.06 & -0.05 & -0.16 & -0.18 & -0.09 & -0.16 & -0.28 & $\mathbf{0 . 3 3}$ \\
\hline Soil Soln_Anion & -0.01 & 0.00 & -0.09 & -0.10 & -0.03 & -0.12 & -0.21 & $\mathbf{0 . 2 9}$ \\
\hline ECe & 0.03 & 0.04 & -0.06 & -0.08 & 0.00 & -0.08 & -0.18 & $\mathbf{0 . 3 1}$ \\
\hline RSC & 0.01 & 0.01 & -0.11 & -0.12 & -0.02 & -0.10 & -0.22 & $\mathbf{0 . 3 5}$ \\
\hline SAR & -0.08 & -0.08 & -0.22 & -0.25 & -0.12 & -0.22 & $\mathbf{- 0 . 3 8}$ & $\mathbf{0 . 4 6}$ \\
\hline
\end{tabular}

Table 7: Correlation between salinity index calculated based on Sentinel 2 bands and soil salinity parameters

\begin{tabular}{|l|c|c|c|c|c|c|c|c|}
\hline \multirow{2}{*}{ Parameters } & \multicolumn{7}{|c|}{ Salinity index based on Sentinel-2 bands } \\
\cline { 2 - 9 } & SI1 & SI3 & SI4 & SI2 & SI5 & SI6 & SI7 & SI8 \\
\hline EC & -0.12 & -0.25 & -0.28 & -0.10 & -0.16 & -0.23 & $\mathbf{- 0 . 4 1}$ * $^{\prime}$ & $\mathbf{0 . 5 1}$ \\
\hline pH & -0.11 & -0.28 & $\mathbf{- 0 . 3 0}$ & -0.10 & -0.15 & -0.29 & $\mathbf{- 0 . 5 2}$ & $\mathbf{0 . 6 3}$ \\
\hline Exch_Ca & 0.18 & 0.25 & 0.26 & 0.16 & 0.21 & 0.25 & $\mathbf{0 . 3 5}$ & $\mathbf{- 0 . 3 0}$ \\
\hline Exch_Mg & 0.00 & -0.05 & -0.07 & 0.01 & 0.00 & -0.03 & -0.12 & 0.22 \\
\hline Exch_Na & -0.12 & -0.30 & $\mathbf{- 0 . 3 3}$ & -0.11 & -0.17 & -0.28 & $\mathbf{- 0 . 5 2}$ & $\mathbf{0 . 6 3}$ \\
\hline Soil Soln_Ca & 0.12 & 0.17 & 0.19 & 0.11 & 0.14 & 0.21 & $\mathbf{0 . 3 0}$ & $\mathbf{- 0 . 2 9}$ \\
\hline Soil Soln_Mg & 0.04 & 0.15 & 0.18 & 0.02 & 0.08 & 0.13 & 0.31 & $\mathbf{- 0 . 4 2}$ \\
\hline Soil Soln_K & 0.06 & 0.08 & 0.08 & 0.06 & 0.05 & 0.13 & 0.13 & -0.08 \\
\hline Soil Soln_Na & -0.07 & -0.18 & -0.21 & -0.06 & -0.11 & -0.17 & $\mathbf{- 0 . 3 3}$ & $\mathbf{0 . 4 1}$ \\
\hline Soil Soln_Carbonates & 0.06 & 0.00 & -0.01 & 0.07 & 0.05 & 0.03 & -0.08 & 0.14 \\
\hline Soil Soln_Cation & -0.05 & -0.15 & -0.17 & -0.04 & -0.08 & -0.13 & -0.27 & $\mathbf{0 . 3 3}$ \\
\hline Soil Soln_Anion & 0.02 & -0.07 & -0.09 & 0.02 & 0.02 & -0.04 & -0.18 & $\mathbf{0 . 3 2}$ \\
\hline ECe & -0.03 & -0.11 & -0.12 & -0.03 & -0.05 & -0.12 & -0.21 & $\mathbf{0 . 3 0}$ \\
\hline RSC & 0.00 & -0.11 & -0.13 & 0.01 & -0.04 & -0.09 & -0.27 & $\mathbf{0 . 3 6}$ \\
\hline SAR & -0.10 & -0.23 & -0.25 & -0.09 & -0.15 & -0.22 & -0.39 & $\mathbf{0 . 4 6}$ \\
\hline
\end{tabular}

Table 8: Linear Regression models for predicting soil salinity parameters from satellite bands reflectance

\begin{tabular}{|c|c|c|c|c|}
\hline Parameter & Satellite & Regression Model & $\mathrm{R}^{2}$ & RMSE \\
\hline \multirow{2}{*}{$\mathrm{pH}$} & Landsat8 & 6.41+15.73(Aerosol/SWIR2)+4.95(Green/NIR)-11.69(Blue/SWIR1) & 0.48 & 0.207 \\
\hline & Sentinel2 & 7.17+8.72((Blue/SWIR2)+1.87(RedEdge2/RedEdge3)-5.44(SI7)-1.0(Blue/RedEdge1) & 0.49 & 0.206 \\
\hline \multirow{2}{*}{$\mathrm{EC}$} & Landsat8 & 0.09+2.02(Aerosol/SWIR2)+0.67(Red/NIR)-7.35(Blue)-0.34(Blue/Green) & 0.33 & 0.07 \\
\hline & Sentinel2 & 0.17-0.94(SWIR2)+1.13(RedEdge1/SWIR1)+1.13(SI7) & 0.30 & 0.07 \\
\hline \multirow{2}{*}{ Sodium } & Landsat8 & -17.04+144.29(Aerosol/SWIR2)+45.66(Green/NIR)-132.28(Blue/SWIR1) & 0.49 & 1.51 \\
\hline & Sentinel2 & -1.59-51.1(SWIR2)+36.39((Blue/SWIR1)+17.28(Green/NIR) & 0.45 & 1.58 \\
\hline
\end{tabular}

\section{DISCUSSION}

Salinization is a multifactor process which influences the physical and chemical soil properties resulting in variation in spectral reflectance properties. Soil texture, roughness, color, mineral composition, organic and moisture content also contribute for significant variations of soil spectral reflectance properties. Soil of the study area is alkali with exchangeable complex dominated with sodium, which destroys soil structure and because of this it showed significant variation in reflectance particularly in SWIR and NIR bands. Numerous studies have illustrated the ability of Vis-NIR (Dwivedi and Rao, 1992; Khan et al., 2005; Fernandez-Buces et al., 2006 and Neild et al., 2007). Atman et al, (2018) and Bannari et al, (2018) have reported good distinction between different soil salinity classes with NIR and SWIR bands of Sentinel 2 satellite data. Soil sampling was done during summer and satellite passes also coincided with same period. During summer no crop was in field and soil surface was vegetation 
free, and because of this reflectance in visible bands as well as salinity indices based on visible bands did not show significant correlation with visible bands. Expressing similar view, Allbed and Kumar (2013) opined that assessment and mapping of soil salinity in areas of densely vegetated soils will yield promising results, whereas on bare soils, the identification of salt based on vegetation indices will not work. Chemical composition and mineralogy of soil contribute significantly for signature in SWIR wavelength and insignificantly in VNIR region (Bannari et al., 2018) whereas optical properties characteristics like colour, brightness and texture etc. contribute to signature in blue and coastal bands (Metternicht and Zinck,2009). Because of this reason rationing of blue or aerosol band with SWIR enhanced the discrimination of categories of alkali soil made up of montmorillonite clay mineral. Previous studies by Allbed et $a l$, (2014) and Fan et al, (2015) also confirmed that the multiple bands transformed into one index will be more sensitive to soil salinity than using a single band. Stepwise regression model developed for soil $\mathrm{pH}, \mathrm{EC}$ and exchangeable $\mathrm{Na}$ using Landsat 8 and Sentinel 2 satellite bands, Band combination and salinity indices as predictor variable showed better prediction accuracy $\left(\mathrm{R}^{2}=0.48-0.49\right)$ for $\mathrm{pH}$ and $\mathrm{Na}$ compared to EC. Following Ali (2011) and Farifteh et al, (2008). soil of study area is slightly sodic $(8.5<\mathrm{pH}<9.0)$ to intensively sodic $(\mathrm{pH}>9.5)$ but less saline. Hence the $\mathrm{pH}$ and Exchangeable $\mathrm{Na}$ model showed better prediction $\left(\mathrm{R}^{2}=0.49\right)$ with $\mathrm{RMSE}$ of 0,21 compared to EC $\left(\mathrm{R}^{2}=0.3\right)$. Soil $\mathrm{pH}$ and exchangeable Na maps developed using above linear regression model closely match with interpolated map developed using ground observations (Figure 3 \&4). Maps developed using Sentinel2 satellite data was finer compared to map developed using Landsat 8 satellite data because of spatial resolution difference.

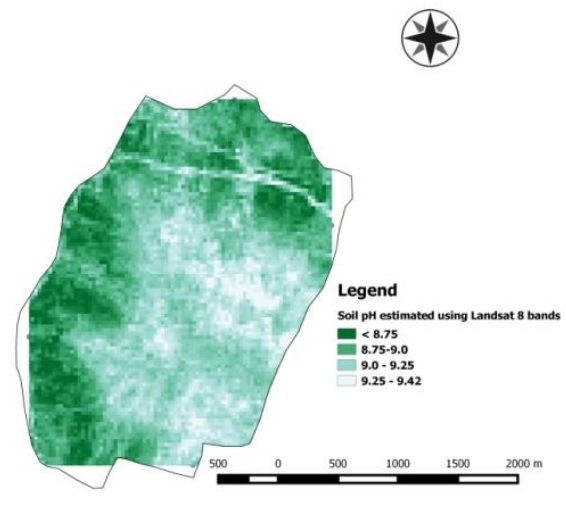

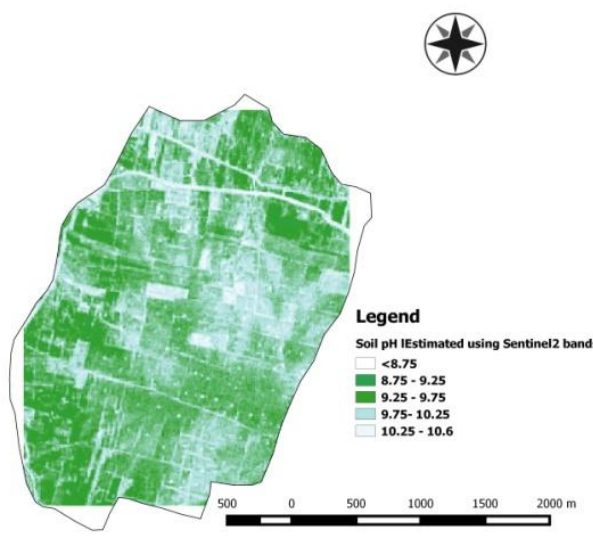

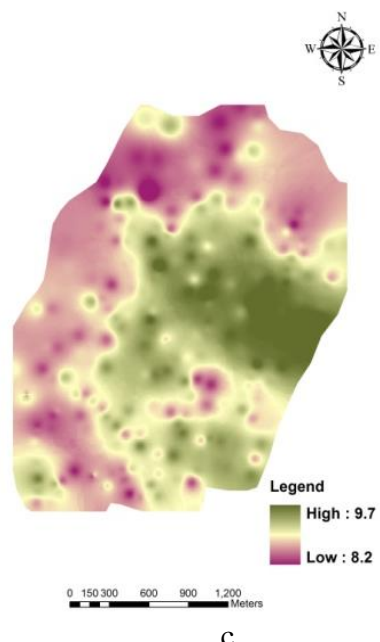

$\mathrm{c}$

Fig3. Map of soil pH predicted using simple linear regression models with Landsat 8 (a), Sentinel 2 (b) bands in comparison with interpolated map (c) using ground observations

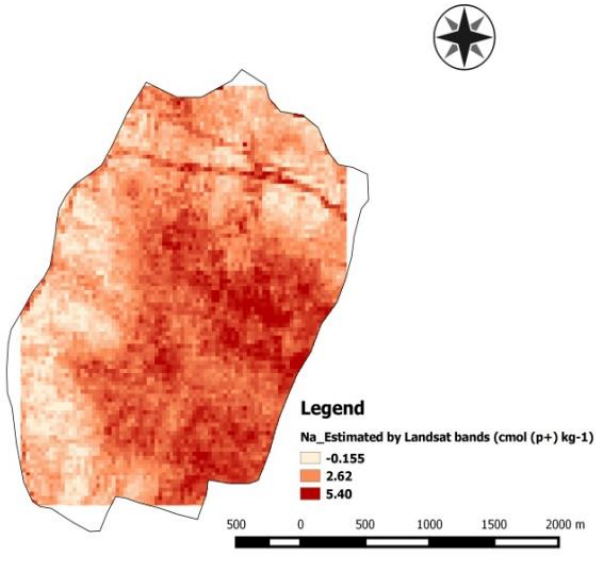

a

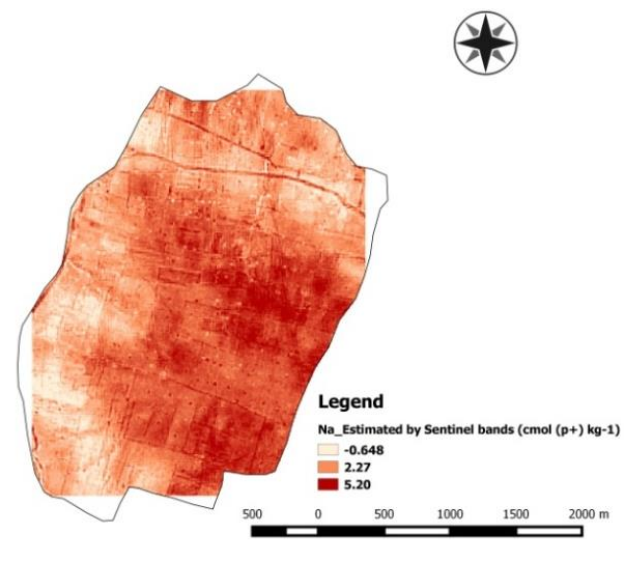

b

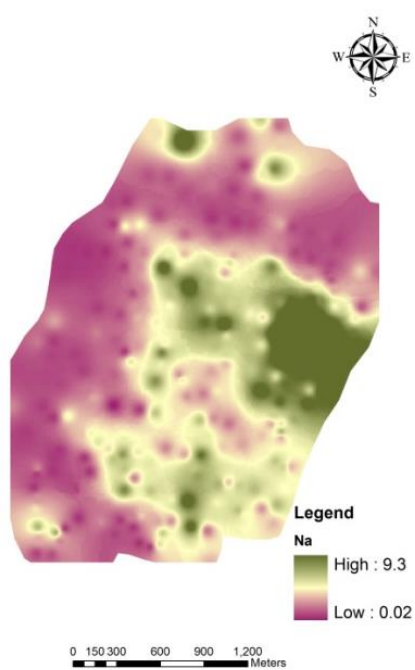

c

Fig4. Map of soil Exchangeable Na predicted using simple linear regression models with Landsat 8 (a), Sentinel 2 (b) bands in comparison with interpolated map (c) using ground observations 


\section{CONCLUSION}

Alkaline sodic soil of study area showed distinct variation in reflectance in SWIR and NIR bands of Landsat 8 and Sentinel 2 satellite. Reflectance in SWIR band showed significant negative correlation with $\mathrm{pH}, \mathrm{EC}$ and exchangeable $\mathrm{Na}$. Rationing of SWIR bands with blue and green bands significantly improved the correlation with soil salinity parameters. All traditional soil salinity index except SI7 and SI8 failed to show correlation with soil salinity parameters. Soil $\mathrm{pH}$ and exchangeable $\mathrm{Na}$ maps developed using simple regression model based on Landsat 8 and Sentinel 2 bands compared well with ground information and will serve as base map for the policy makers.

\section{REFERENCES}

Abbas, A and S. Khan, 2007. Using remote sensing techniques for appraisal of irrigated soil salinity," In: Proceedings of the International Congress on Modelling and Simulation (MODSIM '07), (Eds L. Oxley and D. Kulasiri), Brighton, UK, December 2007, pp. 26322638.

Ali, M.H. 2011.Practices of Irrigation \& on-Farm Water Management, Springer Science + Business Media, LLC: New York, NY, USA, Volume 2, pp. 271-325.

Allbed, A., Kumar, L.and Aldakheel, Y.Y. 2014. Assessing soil salinity using soil salinity and vegetation indices derived from IKONS high-spatial resolution imageries: Applications in a date palm dominated region. Geoderma, 230-231, pp. 1-8.

Atman Ait Lamqadem, Hafid Saber, Abdelmejid Rahimi, 2018. Mapping soil salinity using Sentinel-2 image in Ktaoua oasis (Southeast of Morocco), 7th Digital Earth Summit 2018 (DES-2018). April 17-19, 2018. El Jadida, Morocco.

Bannari, A., Ali El-Battay, Bannari, R. and Hassan Rhinane, 2018. Sentinel-MSI VNIR and SWIR bands sensitivity analysis for soil salinity discrimination in an arid landscape. Remote Sens., 10, pp.855, doi:10.3390/rs10060855

Douaoui, E.K.., Nicolas, H. and Walter, C. 2007. Detecting salinity hazards within a semiarid context by means of combining soil and remote-sensing data, Geoderma., 134(1), pp. 217-230.

Dwivedi, R.S.and Rao, B.R.M.1992. The selection of the best possible Landsat TM band combination for delineating salt-affected soils. Int. J. Remote Sens. 13, pp. 20512058.
Eldeiry, A.A. and Garcia, L.A. 2008. Detecting soil salinity in alfalfa fields using spatial modeling and remote sensing. Soil Sci. Soc. Am. J. 72, pp.201-211.

Fan, X., Liu, Y., Tao, J. and Weng, Y. 2015. Soil salinity retrieval from advanced multi-spectral sensor with partial least square regression. Remote Sens., 7, pp. 488-511.

Farifteh, J., van der Meer, F., van der Meijde, M. and Atzberger, C. 2008. Spectral characteristics of saltaffected soils: A laboratory experiment. Geoderma, 145, pp.196-206.

Fernandez-Buces, N., Siebe, C. , Cram, S. and Palacio, J.L. 2006: Mapping soil salinity using a combined spectral response index for bare soil and vegetation: A case study in the former lake Texcoco, Mexico. J. Arid Environ., 65, pp.644-667

Khan, N. M. Rastoskuev, V. V. Sato, Y. and Shiozawa, S. 2005. "Assessment of hydrosaline land degradation by using a simple approach of remote sensing indicators," Agricultural Water Management, 77(1-3), pp. 96-109.

Metternicht, G. and Zinck, J.A. Remote Sensing of Soil Salinization: Impact on Land Management; CRC Press Taylor and Francis Group: Boca Raton, FL, USA, 2009; $374 \mathrm{p}$.

Mohamed S.B. and Mohamed, B. A. 2017. Spectral unmixing analysis to monitor soil salinity using Landsat imagery for White Nile state Sudan. Malaysian Journal of Remote Sensing \& GIS, 6, pp. 17-26.

Nield, S.J, Boettinger, J.L. and Ramsey, R.D. 2007. Digitally mapping gypsic and natric soil areas using Landsat ETM data. Soil Sci. Soc. Am. J., 71, pp.245-252.

Schmid, T. Koch M. and J. Gumuzzio, 2008. Application of Hyperspectral Imagery to Soil Salinity Mapping," In: Remote Sensing of Soil Salinization: Impact on Land Management, (Eds. G. Metternicht and J. Zinck), CRC Press, Boca Raton, pp. 113-137.

Sumfleth, K.. and Duttmann, R. 2008. Prediction of soil property distribution in paddy soil landscapes using terrain data and satellite information as indicators, Ecol. Indic., 8(5), pp. 485-501.

Taylor, S. 1993. Dry land Salinity-Introductory Extension Notes, 2nd ed.; Department of Conservation and Land Management: Perth, Australia,.

Zhang, T.T., Zeng, S.L., Gao, Y., Ouyang, Z.T., Li, B., Fang, C.M. and Zhao, B., 2011. Using hyperspectral vegetation indices as a proxy to monitor soil salinity. Ecol. Indic. 11, pp. 1552-1562. 